Revue des patrimoines

$31 \mid 2017$

Patrimoines de la santé : essais de définition - enjeux de conservation

\title{
Les bains-douches en Limousin, une hygiène populaire au début du $\mathrm{XX}^{\mathrm{e}}$ siècle
}

Public baths in the Limousin region, popular hygiene in the early twentiethcentury

\section{Colette Aymard}

\section{(2penEdition}

Journals

Édition électronique

URL : https://journals.openedition.org/insitu/14051

DOI : 10.4000/insitu. 14051

ISSN : 1630-7305

\section{Éditeur}

Ministère de la Culture

\section{Référence électronique}

Colette Aymard, «Les bains-douches en Limousin, une hygiène populaire au début du XXe siècle », In Situ [En ligne], 31 | 2017, mis en ligne le 21 février 2017, consulté le 21 septembre 2021. URL : http:// journals.openedition.org/insitu/14051; DOI : https://doi.org/10.4000/insitu.14051

Ce document a été généré automatiquement le 21 septembre 2021.

\section{(i) $\odot$

In Situ Revues des patrimoines est mis à disposition selon les termes de la licence Creative Commons Attribution - Pas d'Utilisation Commerciale - Pas de Modification 4.0 International. 


\section{Les bains-douches en Limousin, une hygiène populaire au début du XX siècle}

Public baths in the Limousin region, popular hygiene in the early twentiethcentury

Colette Aymard

1 En 2006, la Drac et le service des Monuments historiques du Limousin ont décidé de contribuer à une meilleure connaissance de l'architecture $\mathrm{du} \mathrm{xx}^{\mathrm{e}}$ siècle de cette région. Dans le cadre de l'attribution du label «Patrimoine du $x^{e}$ siècle", des travaux de recherche en archives et sur le terrain ont été menés. Parmi les édifices repérés, deux anciens établissements de bains-douches situés à Tulle, en Corrèze, ont suscité des interrogations. Afin de déterminer leur intérêt architectural et d'évaluer leur valeur patrimoniale, un corpus a été réuni et une typologie élaborée. 5 établissements de bains et 30 bains-douches datant du XIX ${ }^{e}$ ou XXe siècle ont été recensés et documentés. Il en existe vraisemblablement d'autres, comme des témoignages oraux ont pu le signaler, mais aucun document n'en fait état.

2 À la suite de cette enquête, un établissement de bains-douches a été protégé au titre des monuments historiques et six autres ont été retenus pour une labellisation au titre du patrimoine $\mathrm{du} \mathrm{xx}^{\mathrm{e}}$ siècle. Un livre intitulé Les bains-douches en Limousin. Architecture et histoire a été publié en 2013 par la Drac et le CAUE du Limousin ${ }^{1}$. Les principaux éléments de cet article en sont tirés.

3 Ce programme de recherche, achevé en 2011, a démontré qu'il s'agissait d'un patrimoine oublié et méconnu. L'histoire des bains-douches correspond chronologiquement à une période très courte. Si le concept est né véritablement en France à la fin du XIX ${ }^{e}$ siècle, la grande majorité des établissements a fermé dès les années 1970 avec la généralisation des salles de bain privées, de même que l'arrivée des machines à laver a entraîné l'abandon progressif des lavoirs publics. 
4 En Limousin, le premier établissement implanté fut celui de Guéret (Creuse) ${ }^{2}$, inauguré en 1909 en présence de René Viviani, ministre du Travail et futur sénateur de la Creuse. Aujourd'hui, sur 30 établissements recensés, un seul fonctionne encore quelques heures par semaine. Il s'agit des bains-douches municipaux situés dans le quartier de la gare des Bénédictins à Limoges.

5 Cette architecture fonctionnelle est pourtant la conséquence directe d'importants progrès dans le domaine de l'hygiène et d'une évolution, voire d'une " révolution », des mœurs. Les préoccupations en matière d'hygiène et de propreté se sont accrues, surtout à partir des années 1870 , jusqu'à devenir un credo sociétal. Elles sont le résultat de bouleversements urbains induits par les révolutions industrielles. Très souvent dues à des sociétés de bienfaisance, en Limousin c'est la caisse d'épargne qui fut le premier maître d'ouvrage à lancer un projet. Elle fut ensuite relayée par les pouvoirs publics et des organismes sociaux tels que mutuelles et sociétés d'habitation à bon marché. Cette diversité de maîtres d'ouvrage a généré des actions et des implantations très disparates sur le territoire de la région.

\section{Le développement de l'hygiène populaire}

6 L'histoire des bains-douches est directement corrélée avec celle des pratiques de l'hygiène du $\mathrm{xx}^{\mathrm{e}}$ siècle. C'est une histoire des pratiques du corps, entre domaine privé et domaine public ${ }^{3}$.

\section{Aux XVIIle-XIXe siècles, un agrément réservé à une minorité}

7 Les usages de l'hygiène se limitent alors souvent à des ablutions rapides et à des pratiques quotidiennes ${ }^{4}$. Ce sont les lavoirs pour les mains, les seaux, les bassines de laiton, les baquets en bois, quelquefois les lavabos de pierre installés dans une niche murale, etc. La propreté des mains est aussi très importante pour des raisons de courtoisie.

8 Par ailleurs, les bains de rivière ont toujours été très prisés et sont, dès le XVIII ${ }^{\mathrm{e}}$ siècle, recommandés pour leurs vertus revigorantes. La philosophie des Lumières, les œuvres de Rousseau, de Diderot et de Voltaire encouragent un retour à l'ordre naturel et à l'exercice physique. De même, les mémoires de Lavoisier sur la physiologie de la transpiration et ses interventions dans le domaine de l'hygiène et de la santé publique concourent à l'essor des bains et des lavages réguliers.

9 Les bains de rivière se développent alors et deviennent aux XVIII ${ }^{\mathrm{e}}$ et $\mathrm{XIX}^{\mathrm{e}}$ siècles de véritables piscines au bord des cours d'eau avec cabines et pontons. Ce sont des établissements dits d'eau courante dont la vocation est d'abord hygiénique ${ }^{5}$. Cette pratique des bains de rivière est attestée par des documents photographiques montrant les bords de Vienne près du pont Saint-Martial à Limoges ${ }^{6}$ et a perduré jusqu'au $\mathrm{xx}^{\mathrm{e}}$ siècle ${ }^{7}$. À Limoges, divers témoignages révèlent que ces bains publics de rivière étaient très appréciés. Outre le pont Saint-Martial, il en existait au pont Neuf ou encore en amont du port du Naveix. L'Almanach Ducourtieux de 1860 évoque cet engouement pour les bains froids de pleine eau :

... Limoges possède encore, sur le bord de la Vienne, un établissement de bains froids qui a conquis de prime-saut la faveur publique. C'est le rendez-vous obligé de tous les amateurs de pleine-eau. Là, plus de vaines distinctions sociales; et sauf de 
rares exceptions, le caleçon de bain y montre les hommes également disgracieux et laids. Quels bons coups de crayons il y aurait là pour Cham $!^{8}$...

10 Malgré ce renouveau pour les plaisirs de la "pleine eau », les pratiques du bain et surtout du bain public sont encore considérées, et ce depuis la fin du Moyen Âge, comme un vecteur de maladies et de contagion ${ }^{9}$. De plus la mauvaise réputation de certains lieux transformés en lieux de prostitution nuit au changement des mentalités. C'est pourquoi aux XVIII ${ }^{\mathrm{e} 10}$ et $\mathrm{XIX}^{\mathrm{e}}$ siècles, les bains publics sont encore majoritairement des lieux de plaisir et de divertissement raffinés ${ }^{11}$. Le bain de piscine ${ }^{12}$, si populaire à Paris à partir du dernier quart $d u \mathrm{XIX}^{\mathrm{e}}$ siècle et vecteur de notions d'hygiène à l'intention des milieux modestes ${ }^{13}$, n'existe pas en Limousin.

11 À Paris, de pittoresques «bains chinois » sont construits, boulevard des Italiens, par Nicolas Lenoir en 1787, lieu de divertissement dans la lignée des luxueux établissements de plaisir anglais dénommés « vauxhalls ».

En Limousin, les « bains chinois » de Tulle (fig. 1), vraisemblablement ouverts dans les années 1820-1830, adoptent une architecture orientaliste s'inspirant d'une pagode : la toiture de ce bâtiment est circulaire et chapeautée par un petit lanternon. Au rez-dechaussée se trouvait une galerie déambulatoire avec une balustrade, protégée par un auvent sur son pourtour. Cette rotonde des bains, située le long de la Corrèze, était construite à l'extrémité d'une promenade, le long des quais. Bordée de haies à proximité de l'édifice, elle bénéficiait ainsi d'une allée ombragée offrant un espace intime et, semble-t-il, alors très prisé des prostituées. Aucun document n'atteste de la présence de cette maison close, destinée à une clientèle bourgeoise, même si cette hypothèse est plus que plausible.

Figure 1

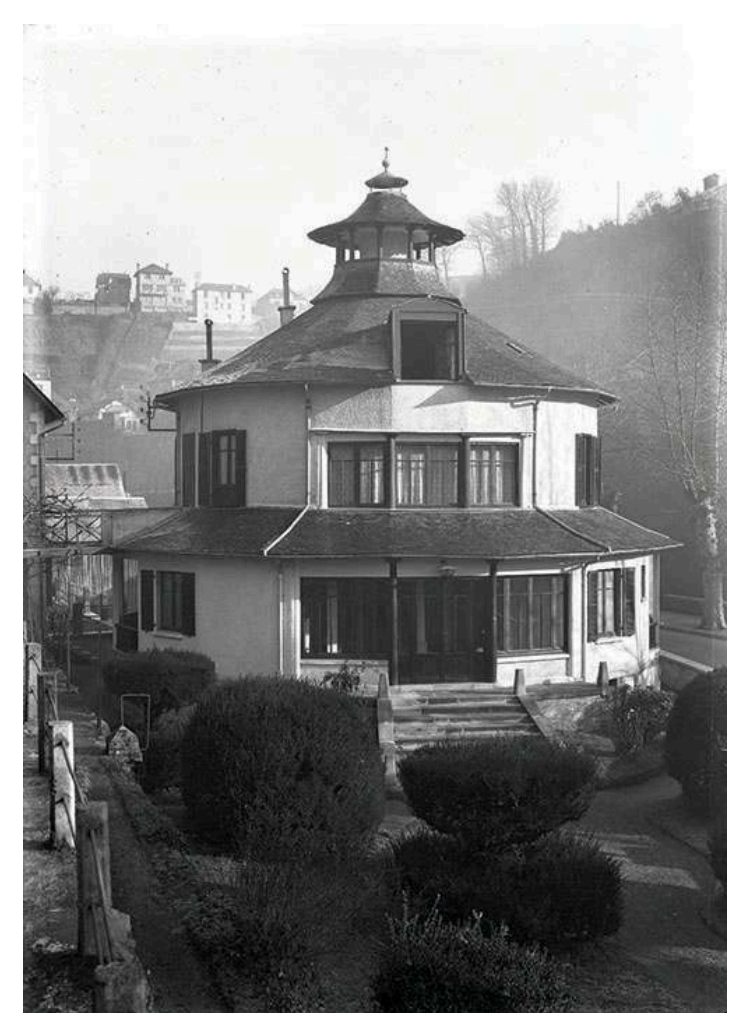

Bains chinois de Tulle, vue générale, fonds Alain et Lucien Durante 1948, 24 Fi 71.

Phot. Archives départementales de la Corrèze. (c) Archives départementales de la Corrèze.

In Situ, 31 | 2017 
13 À Limoges, des bains chinois, aujourd'hui détruits, dispensaient en plein centre-ville des bains de Barèges ou de Vichy, aux vertus thérapeutiques (fig. 2). Il s'agissait d'une entreprise privée commerciale qui était avant tout un lieu de plaisir ${ }^{14}$, offrant à ses clients dans un cadre agréable des soins corporels allant du massage à l'hydrothérapie. Mais ils restaient relativement inaccessibles, de par les prix pratiqués, pour une grande majorité de la population. Les grandes villes et les villes moyennes de la région disposaient elles aussi d'un certain nombre d'établissements privés.

Figure 2

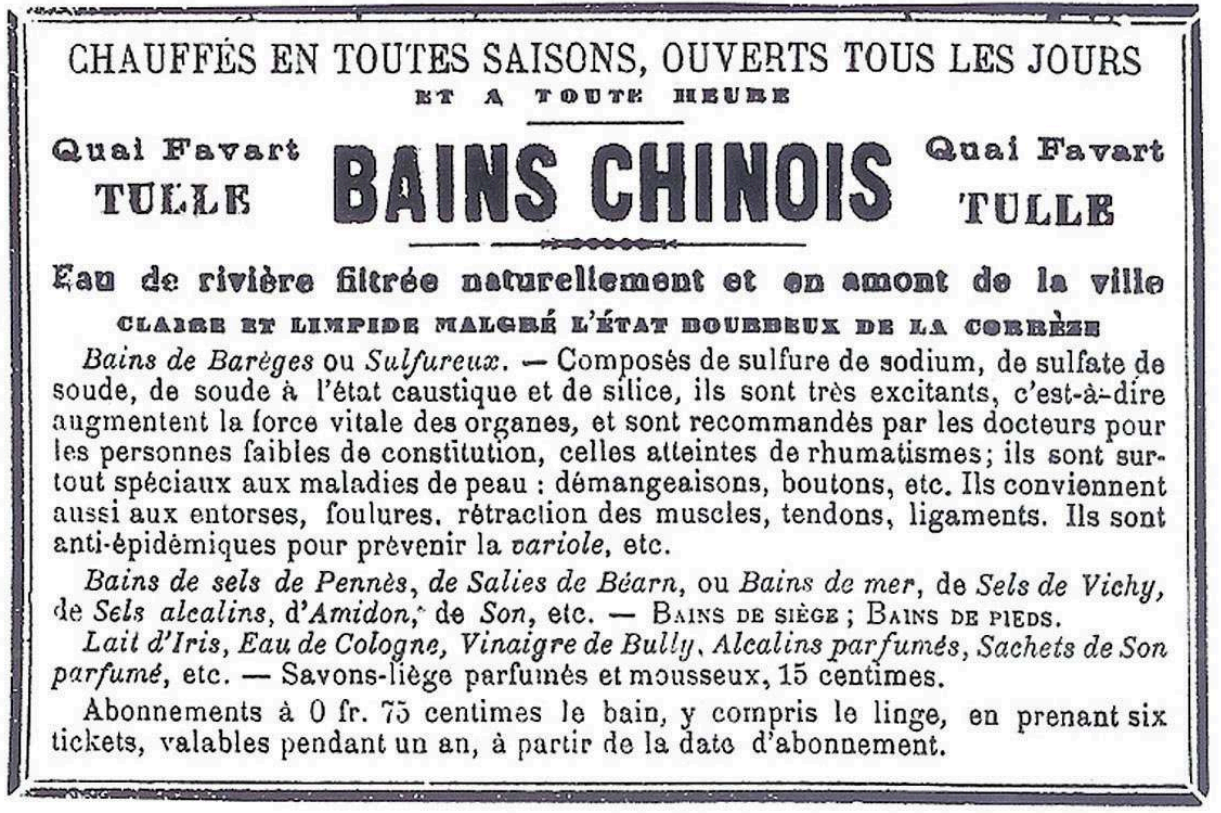

Publicité pour les bains-chinois de Tulle, coll. Société des lettres sciences et arts de la Corrèze.

(c) Société des lettres sciences et arts de la Corrèze.

\begin{abstract}
XIX ${ }^{\mathrm{e}}$ siècle un établissement proposant des bains publics. Situé à proximité de l'école normale, c'était une propriété communale affermée au moins depuis 1883 ; équipé de huit baignoires, il fonctionnait encore en 1930.
\end{abstract}

\section{Le mouvement hygiéniste}

Le $\mathrm{XIX}^{\mathrm{e}}$ siècle fut sur le plan de l'hygiène totalement novateur. Dans les années 1830, David Urquhart, diplomate alors en poste en Orient, importa en Angleterre le bain selon la mode orientale sous la dénomination de «bain turc ». Plusieurs établissements en Europe et en Amérique s'inspirèrent directement des bains turcs londoniens d'Urquhart. Les équipements disponibles étaient très divers : douches simples, douches ascendantes, douches composées, bains de propreté, baignoires simples ou doubles, bains russes de vapeur et de fumigation. Les tableaux de cette époque évoquent cette 
mode avec tous les fantasmes véhiculés par l'imaginaire collectif. Un tableau d'Ingres daté de $1828^{15}$ dénommé La petite baigneuse, représente les bains de vapeur et montre l'extrême raffinement des lieux, la volupté des soins et le luxe des décors orientaux. Ces pratiques d'hygiène mises en scène dans un cadre exotique évoquent le fantasme du harem.

17 Ainsi, au xix ${ }^{\mathrm{e}}$ siècle, les Européens redécouvrent le lavage de tout le corps, jusqu'alors réservé à une élite aristocratique. À côté des bains à l'orientale, à chaleur humide (hammam), se répand aussi la mode des bains russes, à vapeur sèche (sauna).

Le nombre d'établissements dispensant des bains ne reflète pas les profondes mutations de la seconde moitié des $\mathrm{XVIII}^{\mathrm{e}}$ et $\mathrm{XIX}{ }^{\mathrm{e}}$ siècles. Les révolutions politiques et industrielles bouleversent les équilibres sociaux et rendent les infrastructures obsolètes. Insalubrité urbaine et misère sociale vont de pair avec l'industrialisation et l'urbanisation croissante. C'est dans ce contexte que la question de l'habitat et de l'hygiène devient peu à peu une priorité fortement teintée, au XIX ${ }^{e}$ siècle, de moralisme social. Si l'hygiène progresse tout d'abord par le biais de la propreté thérapeutique, l'instauration d'une politique hygiéniste est aussi perçue comme un vecteur de progrès social et doit participer à la création d'un nouvel ordre urbain.

Ce mouvement se développe dans la seconde moitié du XIX siècle et surtout à partir des années 1870 avec le second Empire. Laver le corps est alors conseillé comme soin de propreté et n'est plus seulement une pratique relevant du domaine médical ${ }^{16}$. De très nombreux dictionnaires d'hygiène, comme ceux du docteur Lévy ou d'Ambroise Tardieu $^{17}$, et traités généraux d'hygiène, comme celui du docteur Thouvenel ${ }^{18}$, paraissent à partir de 1848 et se multiplient dans la période 1860-1900. Cette question devient un axe majeur des principes de santé, comme l'affirme le docteur Fonssagrives : «Pourquoi le besoin de se laver n'est-il pas aussi impérieux que celui de respirer? ${ }^{19}$. La propreté est désormais perçue comme un thème essentiel de la politique hygiéniste de l'époque. Et « ...les textes d'hygiène [...] insistent au début du XIX ${ }^{e}$ siècle sur quelques renouvellements de pratiques : la promotion du savon par exemple. Celui-ci gomme et dissout la crasse. Il « purifie $» . . . »^{20}$. Un fait illustre ce mouvement de société et atteste l'usage courant du savon : l'entreprise de savons de Marseille Le chat est créée en 1853.

C'est dans ce contexte que 'autorité publique engage une politique sanitaire en faveur des classes populaires. En 1878, le premier Congrès international d'hygiène se tient à Paris. L'État déclare l'hygiène d'intérêt public par la loi du 5 avril 1884. Les prisons ${ }^{21}$, asiles de nuit et casernes bénéficient très rapidement de cette politique de prophylaxie hygiénique. Les lycées et les crèches sont aussi des lieux propices à une éducation populaire en matière d'hygiène et à l'apprentissage de nouveaux comportements sur le plan sanitaire ${ }^{22}$. Dès 1848 , Hippolyte Carnot, ministre de l'Instruction publique, avait d'ailleurs institué une commission d'hygiène des lycées et fixé des normes sanitaires. Ces deux mesures sont à l'origine de la mise en place progressive de bains de pieds et de salles de douches dans les lycées. Malgré de nombreuses mesures et une action très interventionniste des médecins hygiénistes, la pratique de l'hygiène à l'école reste limitée en 1900. Des "manuels d'éducation " sont publiés pour vulgariser les règles fondamentales de l'hygiène. La littérature enfantine contribue à cette diffusion : dans Les malheurs de Sophie ${ }^{23}$ de la comtesse de Ségur, la petite fille du Second Empire apprend les bases de l'hygiène corporelle et les reproduit sur sa poupée ${ }^{24}$.

21 Ainsi, peu à peu, le laisser-faire libéral n'est plus jugé acceptable et dès la seconde moitié $\mathrm{du} \mathrm{XIX}^{\mathrm{e}}$ siècle et le début $\mathrm{du} \mathrm{xx}^{\mathrm{e}}$ siècle, l'hygiénisme et la propreté intègrent la 
sphère publique jusqu'à devenir des valeurs sociétales, considérés comme des vecteurs favorables au progrès, à la moralisation des mœurs et à l'ordre social. L'hygiène populaire est au cœur des débats.

\section{Une lente évolution des mentalités}

Les progrès de l'hygiène sont cependant très tributaires des valeurs morales qui ont alors cours. Or, au XIX ${ }^{e}$, les bains publics et les pratiques de la Grèce antique ne sont plus de mise: «... le bain est encore souvent une pratique immorale...» comme le déclarent en 1852 les membres du conseil d'hygiène de Nantes ${ }^{25}$. Il est en effet souvent assimilé dans les esprits aux femmes de mauvaise $v^{26}{ }^{26}$ et l'usage, au milieu du XIX ${ }^{e}$ siècle, tend plutôt à favoriser un lavage partiel (fig. 3). Même si le nettoyage du corps entier est de plus en plus recommandée ${ }^{27}$ par les médecins à partir du début du $\mathrm{xx}^{\mathrm{e}}$ siècle, le bain quotidien est déconseillé.

Figure 3

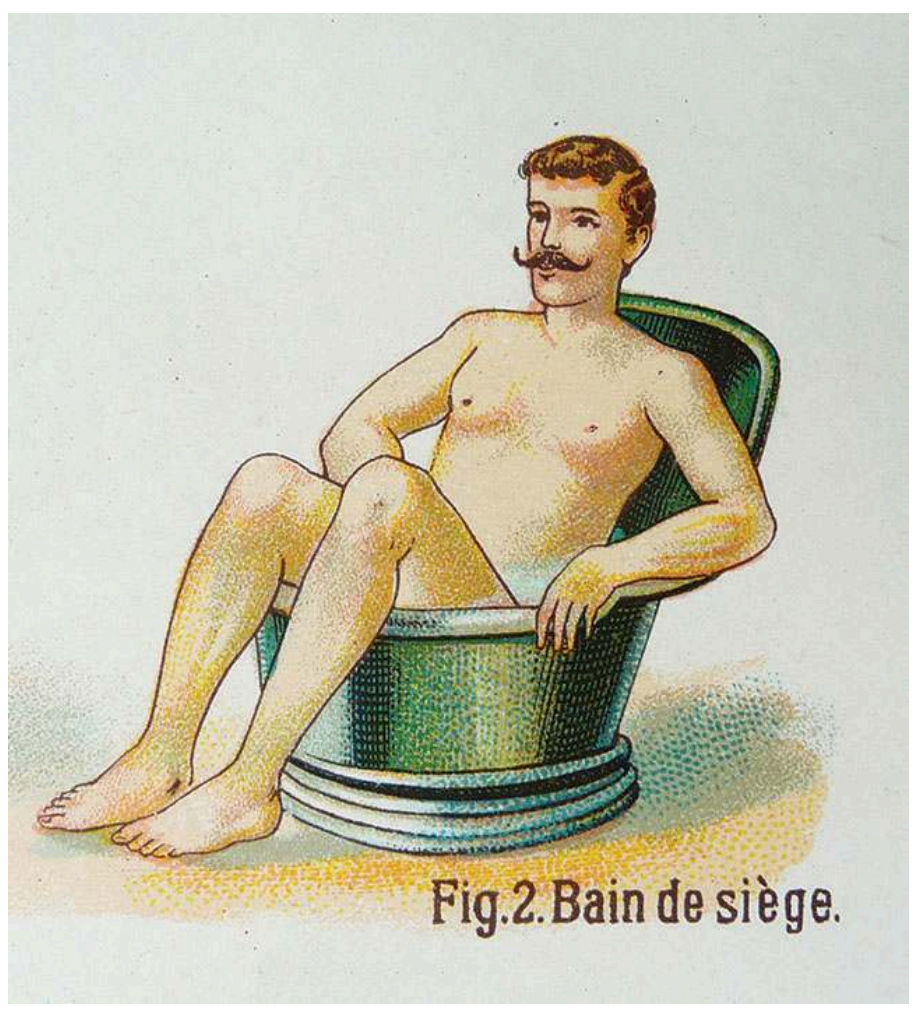

Bain de siège. Friedrich Eduard Bilz, " La médication naturelle. Traité et aide mémoire de médication et d'hygiène naturelle », 1898, T. 2, chap. «bains de vapeur », p. 1744 bis.

En fait, les préconisations en terme d'hygiène varient selon les classes sociales ${ }^{28}$. Trois ouvrages, de la baronne Staffe, de la comtesse de Gencé2 ${ }^{29}$ et de la comtesse de Tramar ${ }^{30}$, fournissent au $\mathrm{XIX}^{\mathrm{e}}$ siècle les règles de savoir-vivre qui sont autant de signes distinctifs et d'appartenance sociale: pour les plus pauvres, la norme admise est d'un bain mensuel avec des ablutions quotidiennes de la bouche; pour les plus favorisés, un bain hebdomadaire est conseillé. La baronne Staffe, très novatrice, recommande, dans son manuel de savoir-vivre Usages du monde ${ }^{31}$ publié en 1891 et destiné aux jeunes filles et aux dames, un bain deux fois par semaine et chaque jour la pratique du « tub $b^{32} »$. 
En 1899, un rapport établi par une commission émanant du conseil municipal de Vincennes ${ }^{33}$ conclut «la douche peut être mauvaise à la santé des enfants dont les organes sont en formation; elle peut provoquer des affections nerveuses et développer les maladies cardiaques. Enfin elle ne nettoie pas, car la douche décolore la crasse sans l'enlever... ». La douche est ainsi souvent réputée dangereuse $\mathrm{e}^{34}$ et inefficace à entretenir la propreté. Elle était pratiquée, de manière brutale, pour calmer les états de folie : « ... La douche comporte le jet violent d'une masse d'eau plus ou moins divisée dont l'énergie se communique en partie à la peau qui se déplace, se déforme et vibre pour ainsi dire sous l'attaque... $»^{35}$. La douche est avant tout perçue comme une pratique hydro-thérapeutique, notamment par les couches populaires ${ }^{36}$. Des ouvrages sur l'hygiène largement diffusés, comme celui du professeur Debove et du docteur Plicque $^{37}$, soulignent le danger des douches : « les douches ne constituent pas un simple moyen d'hygiène et de propreté ; elles entraînent de vives réactions. Elles ne doivent pas être prises au hasard et sans avis médical... ».

\section{Les bains-douches, une œuvre sociale}

À la fin du xix ${ }^{\mathrm{e}}$ siècle on dissocie donc douches et bains-douches : le docteur Merry Delabost expérimente en 1872 ce dernier procédé dans les prisons de Rouen afin d'améliorer l'hygiène des prisonniers. Malgré ses nombreux articles, cette pratique ne parvient pas à se développer en France ${ }^{38}$. Elle est pourtant simple et économique : il s'agit d'une simple pluie d'eau chaude intermittente. Ces bains-douches de propreté sont recommandés par les médecins hygiénistes, contrairement au bain ou à la douche. Ils ont l'avantage d'associer le lavage des corps à un temps relativement court, avec une quantité d'eau réduite. Économie et rationalité en sont les maîtres mots. Les établissements de bains-douches sont, dès l'origine, conçus comme des lieux de propreté populaire qui doivent contribuer au développement de la morale et de l'ordre public. Ainsi, lorsqu'une société de bienfaisance tente de créer les premiers établissements à Limoges, en 1907, elle expose dans son projet que «...Les bainsdouches donnent la propreté à bon marché, combattent l'alcoolisme, évitent la tuberculose, arrêtent les épidémies, contribuent à l'hygiène morale par l'hygiène physique... $»^{39}$. À travers l'hygiène, les élites locales tiennent à moraliser la société et plus particulièrement la population ouvrière. La morale du corps est étroitement liée à la morale des esprits (fig. 4). 


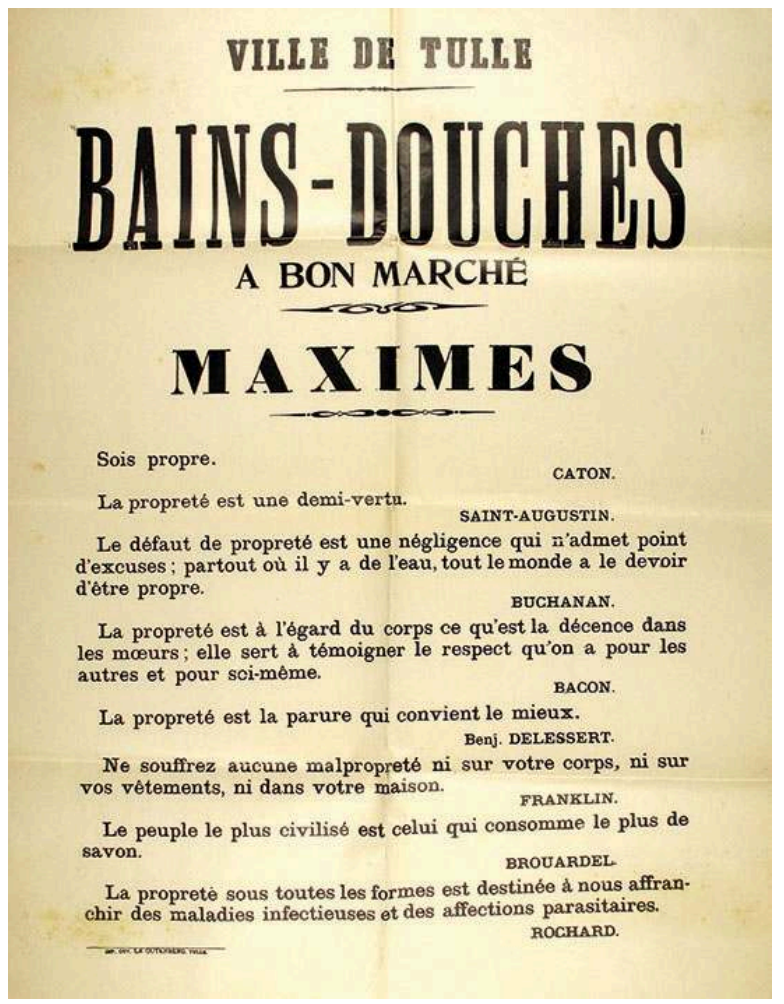

Affiche située auparavant dans les bains-douches à bon marché, 201830.

Phot. Archives départementales du Cher. ( ) Archives départementales du Cher.

Les philanthropes et les sociétés de bienfaisance sont les premiers, en France, à fonder des bains-douches populaires en tant qu'œuvre sociale. À l'initiative de Charles Cazalet, six médecins créent à Bordeaux en 1893 les premiers bains-douches populaires ${ }^{40}$. C'est un succès immédiat, souvent pris comme modèle: Rouen ${ }^{41}$ et Paris en $1898^{42}$, La Rochelle en $1902^{43}$, puis Troyes, Lyon, Belfort, etc. À Limoges, seule une société de bienfaisance a tenté de mettre en place un projet de bains-douches qui n'a pas abouti. Devant le succès de ces premières initiatives philanthropiques, l'État décide d'intervenir en faveur de leur développement. La loi Strauss, du 12 avril 1906, attribue des facilités d'emprunt pour la construction de bains-douches ${ }^{44}$ et contribue ainsi à leur essor. L'hygiène devient alors officiellement publique.

\section{Des promoteurs divers, des motivations différentes}

En Limousin, la diversité architecturale des établissements de bains-douches est liée au maitre d'ouvrage : la construction des bains-douches est représentative des ambitions, des moyens et des types d'équipement souhaités par ses différents promoteurs.

\section{Des mutuelles soucieuses de leurs adhérents}

Les mutuelles créent des bains-douches. En effet, face à la misère grandissante, l'État est très démuni. Les premières mutuelles sont apparues dans les années 1830 en Grande-Bretagne ${ }^{45}$, en lien avec la révolution industrielle. En France, un décret 
reconnaît les sociétés de crédit mutuel en 1852 . Si elles restent apolitiques ${ }^{46}$, la création de coopératives devient légale en 1867 mais c'est seulement à partir de la charte de la mutualité de 1898 que les mutualistes peuvent mener librement leurs activités. Les mutuelles suscitent en effet bien souvent la méfiance des pouvoirs publics qui craignent la création d'un contre-pouvoir et la montée des contestations sociales. Ce sont donc des associations "de prévoyance », créées pour prémunir les adhérents des risques importants de la vie tels que maladies, accidents, handicaps, vieillesse ou encore décès. Pour pouvoir en bénéficier, l'adhérent doit s'acquitter d'une cotisation ${ }^{47}$ qui est souvent forfaitaire. Les premières mutuelles sont d'origine privée et regroupent en général des travailleurs exerçant la même profession, de nature donc corporatiste. L'affiliation est librement consentie et n'est pas obligatoire. À Limoges, la mutualité limousine regroupe majoritairement des ouvriers. Ces structures répondent aux besoins sociaux en organisant une nouvelle forme de solidarité (distribution d'aliments, garderie des enfants, médecine à prix réduits, caisse en cas de décès ou d'invalidité, etc.). C'est dans ce cadre de prévention et d'amélioration de l'hygiène publique de leurs sociétaires que les mutuelles sont également à l'origine de bains-douches, réservés à leurs seuls adhérents.

Les sociétés mutuelles de Limoges sont très dynamiques et cherchent à faire profiter leurs adhérents des récents progrès sanitaires en fondant le deuxième établissement du Limousin. Les bains-douches mutualistes à bon marché (fig. 5) sont construits en 1910, à l'angle de la rue Turgot et de la rue des combes, en plein centre-ville. Leur architecture se veut de prestige et adopte un style néo-classique ${ }^{48}$.

Figure 5

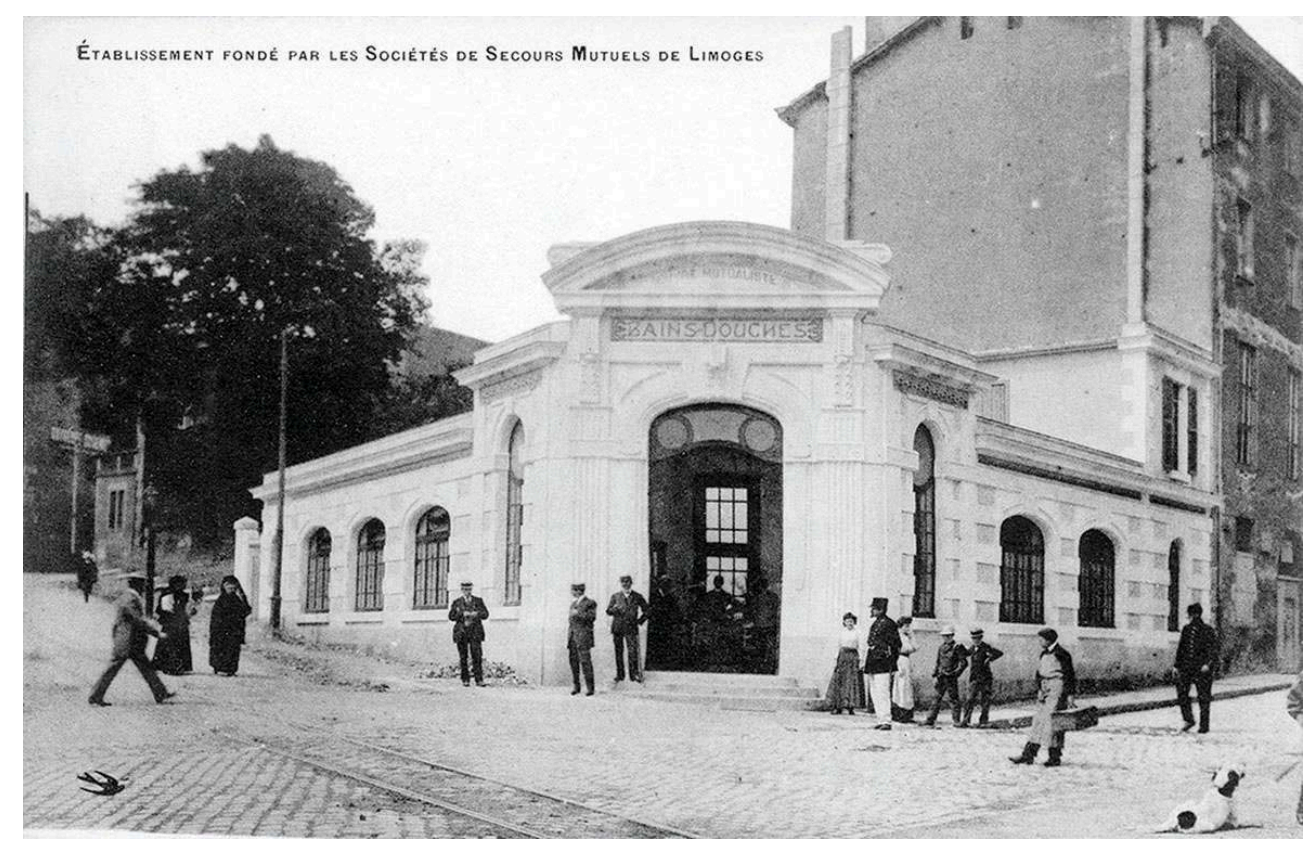

Bains-douches mutualistes à bon marché de Limoges, à l'angle des rues Turgot et des combes. Photothèque Paul Colmar.

(C) Photothèque Paul Colmar.

Des municipalités attentives à la vie quotidienne 

institutions qui accueillent des enfants sont les premières à bénéficier de ce mouvement. C'est, par exemple, en Gironde, la création en 1898 de bains-douches dans la crèche de La Bastide à Bordeaux et dans les écoles maternelles de Caudéran et de Talence la même année. Les villes sont de plus en plus nombreuses à souhaiter l'ouverture de bains-douches municipaux pour une clientèle plus large. À partir des années 1920-1930, de nouveaux textes réglementaires élargissent les possibilités d'intervention communale «dans la réalisation d'améliorations urbaines ${ }^{49}{ }$; les communes peuvent emprunter pour s'assurer les moyens de leur politique. Les réalisations sont laissées à l'initiative des communes et l'État adopte une position noninterventionniste. La mise en place des réseaux d'eau, d'égouts ou d'électricité est également l'occasion pour de nombreuses villes de s'équiper de bains-douches.

e architectural de ces établissements varie selon les motivations qui ont présidé à leur création. Si les municipalités cherchent à affirmer le statut de bâtiment communal, le style reproduit l'architecture propre aux mairies, comme à Corrèze, où l'architecture régionaliste est empreinte d'une grande modestie. Cet édifice, construit en 1939, regroupe plusieurs services publics: foyer familial des campagnes, mairie, bureau de poste, bureau de justice de paix, bains-douches, lavoirs et remises pour le corbillard et la pompe à incendie. Il est surmonté d'un clocheton pour manifester l'expression du pouvoir local, face à l'église (fig. 6).

Figure 6

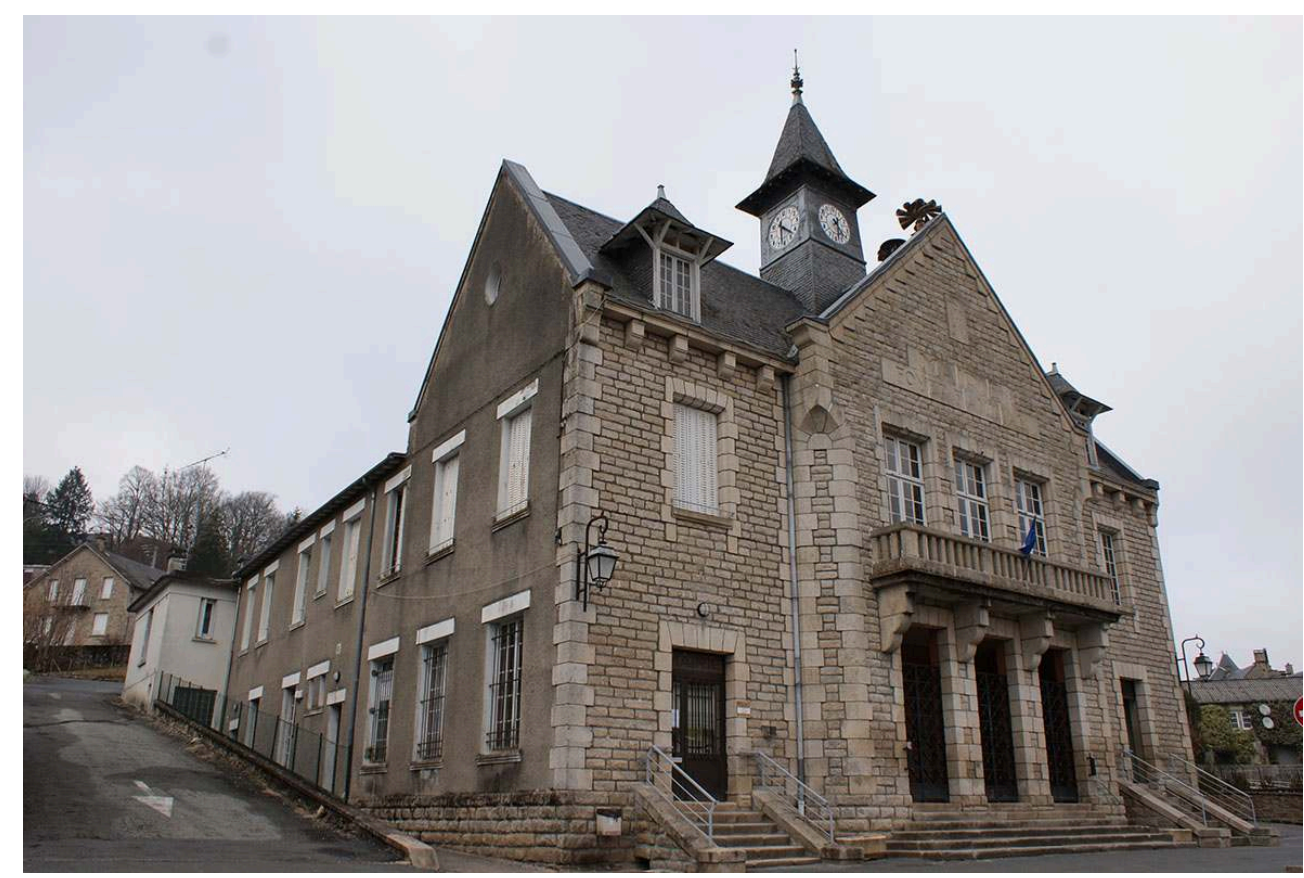

Façade du bâtiment communal, commune de Corrèze.

Phot. Aymard, Colette, 2010. (c) Colette Aymard.

Quand les bains-douches ont été édifiés dans le cadre d'un programme d'aménagement urbain, ils sont représentatifs, avec la mairie, du pouvoir municipal. On peut en citer deux exemples: la commune de Saint-Yrieix-la-Perche (Haute-Vienne) aménage en 1939 des bains-douches pour compléter le nouveau quartier administratif, à l'extérieur du centre médiéval. La mairie s'y était installée en 1901, c'est au tour de la poste en 
1950. L'architecture est fonctionnelle, centrale, et adopte une composition symétrique. À Chamberet (Corrèze), lors de l'aménagement du centre-ville, autour d'une place publique et d'un marché, on construit d'un côté de la place un grand portique donnant sur une galerie et de l'autre, des bains-douches. Cet aménagement imposant est atypique pour une petite commune rurale isolée de la Corrèze (fig. 7).

Figure 7

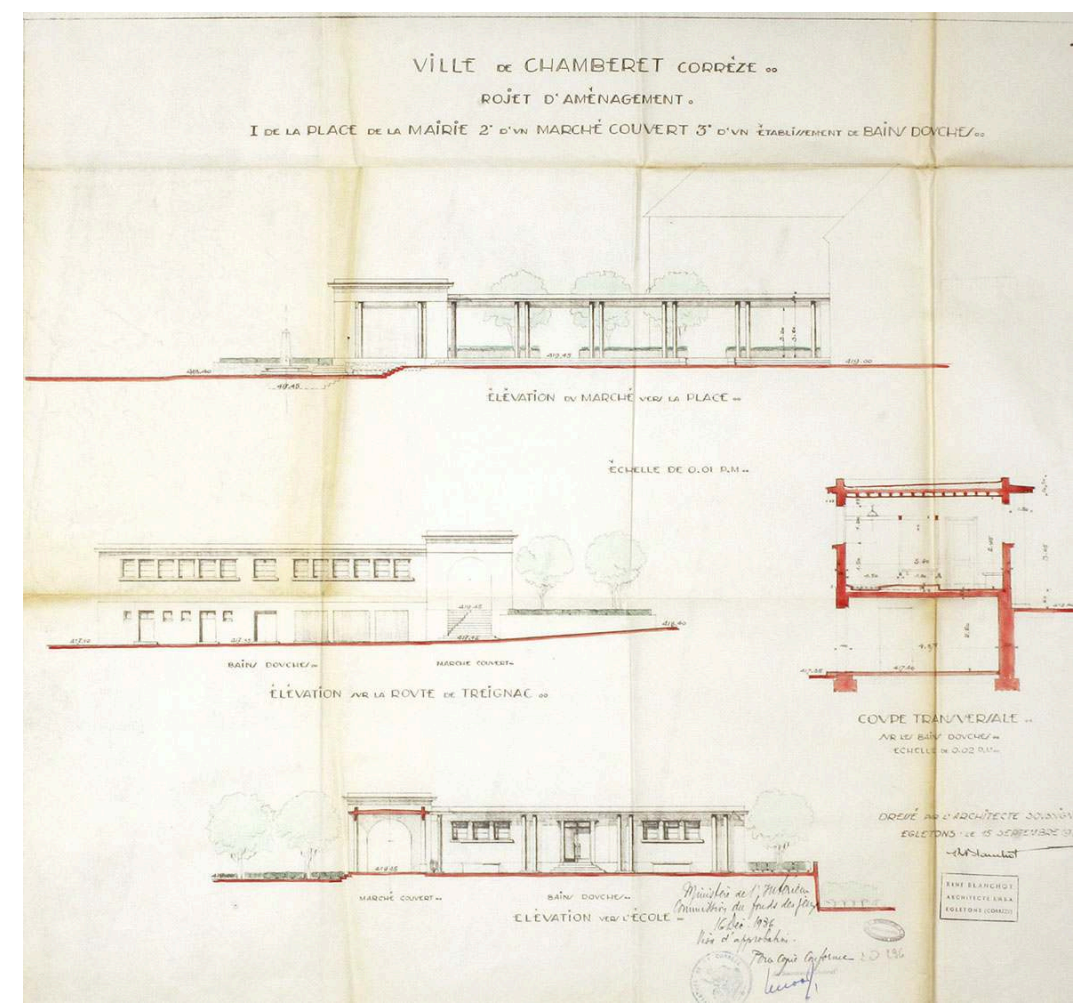

Plans du projet des bains-douches dressé par l'architecte, commune de Chamberet, 202960.

Phot. Archives départementales du Cher. @ Archives départementales du Cher.

Le troisième type architectural est caractérisé par des édifices dont l'architecture se veut emblématique. Afin de marquer les esprits, le style est encore plus affirmé : les bains-douches de Tulle, au pont de la Barrière ${ }^{50}$, construits en 1910, reprennent le style Art nouveau (fig. 8) ; l'établissement de Bort-les-Orgues (Corrèze) ${ }^{51}$, ouvert en 1934, adopte un style emprunté à l'architecture moderne et à l'Art déco ; les bains-douches de Chénérailles (Creuse) ${ }^{52}$, datés de 1959 et munis d'une laverie, sont typiques de l'architecture fonctionnelle des années 1950. 


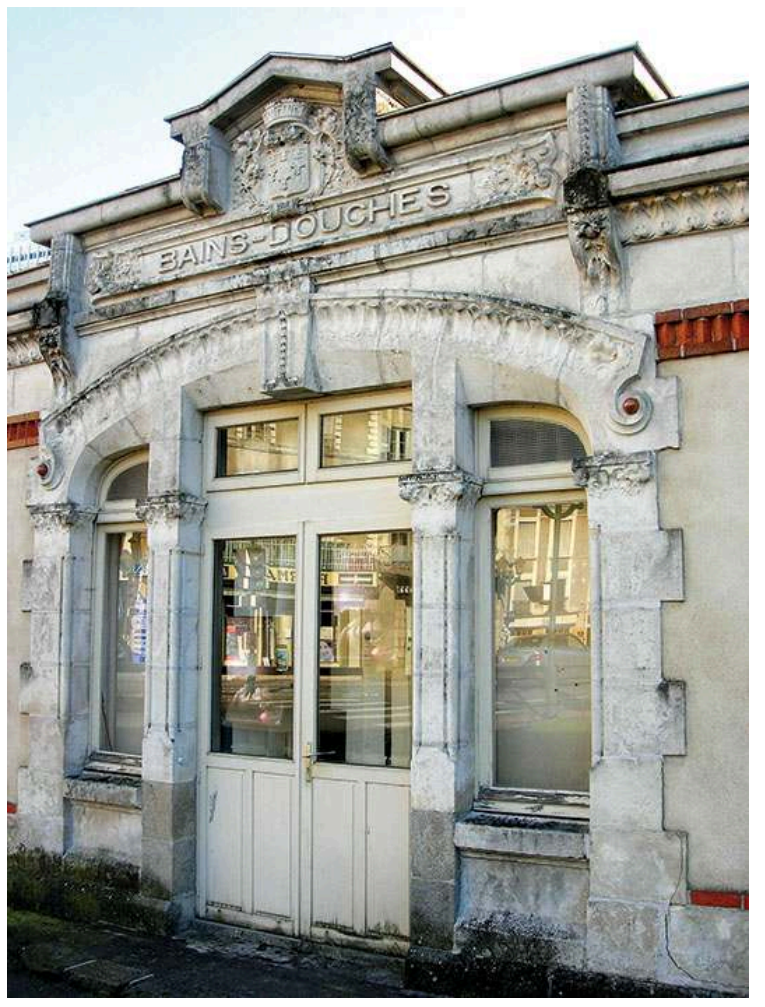

Façade principale des bains-douches de Tulle.

Phot. Pradoux, Josiane, 2010. (c) Josiane Pradoux.

Le quatrième style reprend, pour les communes situées en milieu rural, l'architecture des pavillons de style néo-normand en vogue en Limousin dans les années 1930, comme à Boussac (Creuse), Égletons (Corrèze) ou Grand-Bourg (Creuse).

Le cinquième type architectural peut s'observer dans les bains-douches communaux plus tardifs : à partir des années 1950, la notion d'hygiène évolue; ils sont associés le plus souvent à la pratique sportive, comme c'est le cas pour le nouvel établissement de Bellac (Haute-Vienne) $)^{53}$, installé à proximité du stade.

\section{Des sociétés d'HBM au service des habitants}

Les bains-douches des sociétés d'habitations à bon marché (ou HBM) représentent la troisième catégorie de maître d'ouvrage. Avec la loi Bonnevay de 1912, l'État favorise la création d'offices d'HBM par les collectivités locales. Dans ces ensembles d'habitations, il souhaite favoriser l'intégration sociale et une vie plus harmonieuse par la mise en place d'équipements et de services tels que bains-douches, crèche, commerces, espaces de jeux, etc. L'office public des habitations à bon marché (OPHBM) fondé à Limoges en 1920 crée de nombreuses cités populaires dans l'entre-deux-guerres en y intégrant un établissement de bains-douches. Les cités des Marronniers et de Montjovis en sont des exemples : construites en 1913, elles bénéficient de cabines de bains-douches installées au rez-de-chaussée de l'immeuble. Le bâtiment adopte généralement un style industriel et reprend l'architecture des entrepôts. Ce service social, installé à moindre coût au sein même des cités doit être fonctionnel et pratique pour les résidents. La cité des Coutures (fig. 9), plus importante, aménagée entre 1930 et 1932, bénéficie d'une 
gamme d'équipements plus large, regroupant au centre une crèche, des bains-douches et des lavoirs. Le style est d'inspiration balnéaire, avec une galerie à balustrade, une terrasse, des colonnes, et est disposé en position légèrement dominante. Fermés en 1971-1972, les bains-douches ont été détruits.

Figure 9

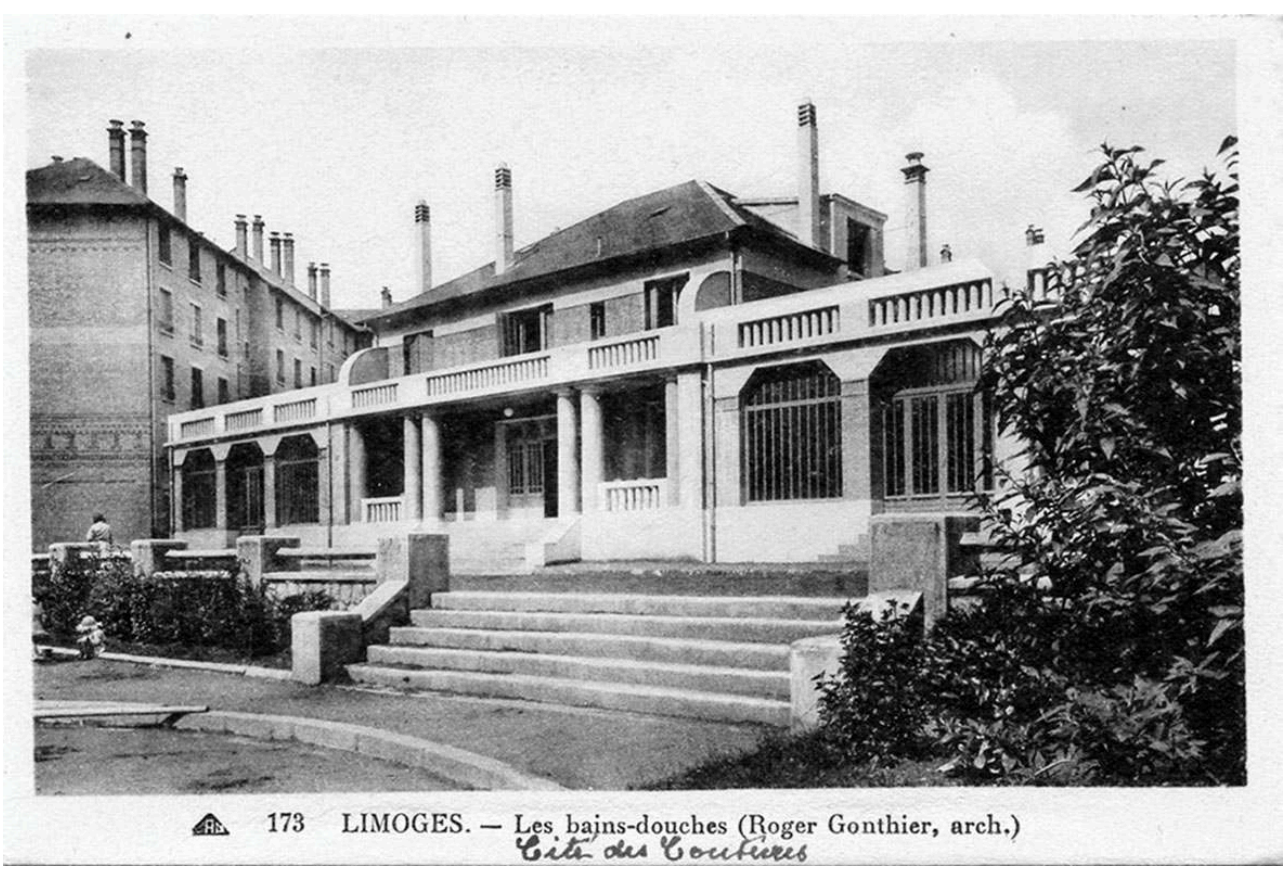

Bains-douches des Coutures, Limoges. Photothèque Paul Colmar.

(c) Photothèque Paul Colmar.

\section{Des caisses d'épargne assurant une mission d'utilité publique}

Enfin, de très nombreux bains-douches sont construits par les caisses d'épargne. Les premiers bains-douches populaires établis en France en 1893, à l'initiative de Charles Cazalet, le sont par une œuvre philanthropique qui réunit six médecins bordelais et le maire de la ville, Adrien Bayssellance. Ce premier établissement de bains-douches, appelé «l'œuvre bordelaise des bains-douches à bon marché ", est dû à l'action de philanthropes, alors qu'il aurait pu être financé par les caisses d'épargne : l'œuvre bordelaise avait sollicité une aide de $20000 \mathrm{~F}$ mais ce financement lui fut refusé. Charles Cazalet relate ainsi la réponse de la caisse d'épargne locale : «...Notre demande fut d'abord accueillie par des rires, qui nous furent ensuite traduits par cette réponse sans réplique : «L'argent sacré de la Caisse d'épargne ne peut servir à faire baigner des

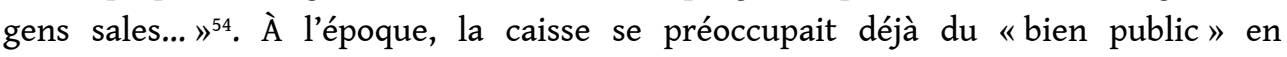
subventionnant essentiellement des habitations à bon marchées, des logements économiques ou encore des restaurants à bon marché ${ }^{56}$. Charles Cazalet s'interrogea alors sur la nécessité de créer une "...société de crédit destinée à favoriser le développement des bains-douches à bon marché... ». Il estima finalement qu'il faudrait " ...qu'une loi nouvelle vienne autoriser la Caisse des dépôts et consignations à prêter les fonds nécessaires à la Société de crédit des habitations et des bains-douches... ${ }^{57}$. L'État répondit favorablement à cette demande, afin d'encourager le développement de 
ces constructions, par la loi du 12 avril 1906 qui autorisait les caisses d'épargne à intervenir dans le secteur social. Elles contribuaient en effet à la protection de l'épargne populaire, mais dès la seconde moitié $\mathrm{du} \mathrm{XIX}^{\mathrm{e}}$ siècle, elles participaient à de nombreuses opérations à vocation sociale comme le financement de logements et restaurants à bon marché dès 1889 , ou encore, à partir de 1906, le financement de jardins ouvriers et la création de bains-douches. L'État devenait par cette jurisprudence un acteur financier indispensable et reconnaissait la nécessité de soutenir la création de nouveaux établissements pour développer l'hygiène; avec cette loi, l'hygiène était officiellement de la responsabilité des pouvoirs publics. C'est pourquoi les caisses d'épargne, reconnues «établissements privés d'utilité publique ", depuis la loi du 5 juin $1835^{58}$, devinrent à partir de 1906 le principal organisme de prêt pour la création d'établissements de bains-douches. Elles contribuaient d'ailleurs par ce biais à développer localement des actions de prévoyance, d'hygiène et d'assistance et s'imposaient comme un acteur essentiel de la vie locale. Avec la loi de 1906, les caisses d'épargne assumaient pleinement la mission d'hygiène publique, au titre de leurs missions d'utilité publique. C'est le conseil des directeurs de la caisse d'épargne qui décida en 1907 de la construction du premier établissement de bains-douches à Amiens.

Désormais, ces organismes étaient légalement autorisés à acquérir ou à financer des bains-douches. Ils prêtaient donc de l'argent aux communes ou en assuraient la construction et la gestion au nom du bien public. Forts de cette compétence, les caisses d'épargne publièrent un guide, La manière de prendre un bain-douche, dont l'existence démontre à quel point cette pratique était alors considérée comme novatrice en matière d'hygiène.

Deux styles architecturaux sont choisis. Les bains-douches de Guéret (fig. 10) tout comme ceux de Bourganeuf (Creuse), datés de 1912, sont représentatifs d'un style urbain plutôt bourgeois ou cossu. Le bâtiment d'Aubusson (Creuse) ${ }^{59}$, installé dans un quartier populaire en 1927 pour compléter un nouvel ensemble de huit «maisons ouvrières à bon marché ", adopte le style des années 1930 et devient un équipement indispensable de ce quartier. Il se veut original par la présence, en façade, d'un attique d'un mètre quarante de haut ${ }^{60}$, élément aujourd'hui disparu. 
Figure 10

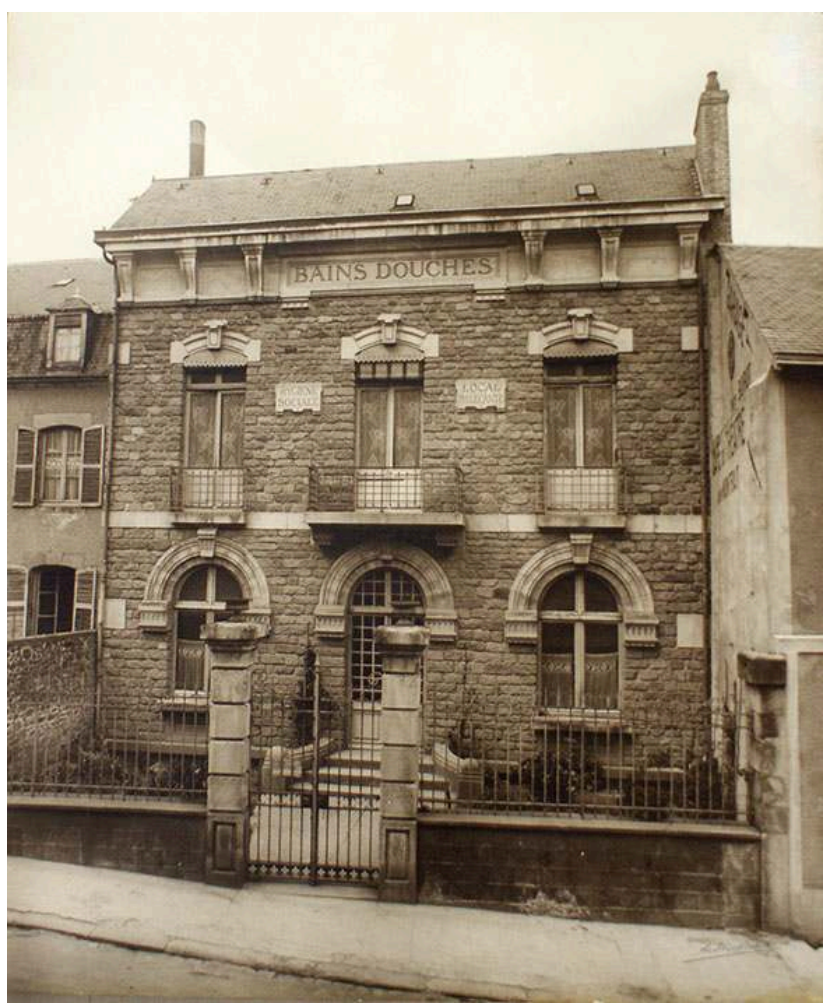

Bains-douches de Guéret, vue générale sur le bâtiment et de sa façade sur rue. Arch. de la caisse d'épargne d'Auvergne et du Limousin.

(c) FNCE.

En milieu rural, comme à Bonnat (Creuse) ou à Dun-le-Palestel (Creuse), la caisse d'épargne construit dans un style pavillonnaire cossu alors à la mode en Limousin qui copie celui des maisons néo-normandes avec toiture débordante et aisseliers en bois (fig. 11). Ces établissements ont une double fonction, à la fois caisse d'épargne et bainsdouches. Ces derniers sont souvent disposés à l'arrière du bâtiment. L'intérieur observe presque toujours le même plan: une partie pour les hommes, une partie pour les femmes. Les cabines de douche sont disposées le long des murs extérieurs et desservies par un couloir central. Une cabine fait environ 2,5 mètres carrés et se divise en deux parties: un déshabilloir et un espace de douche de la même surface. L'utilisateur ne peut pas rester plus de 15 à 20 minutes, il bénéficie d'une pluie intermittente, par mesure d'économie. 
Figure 11

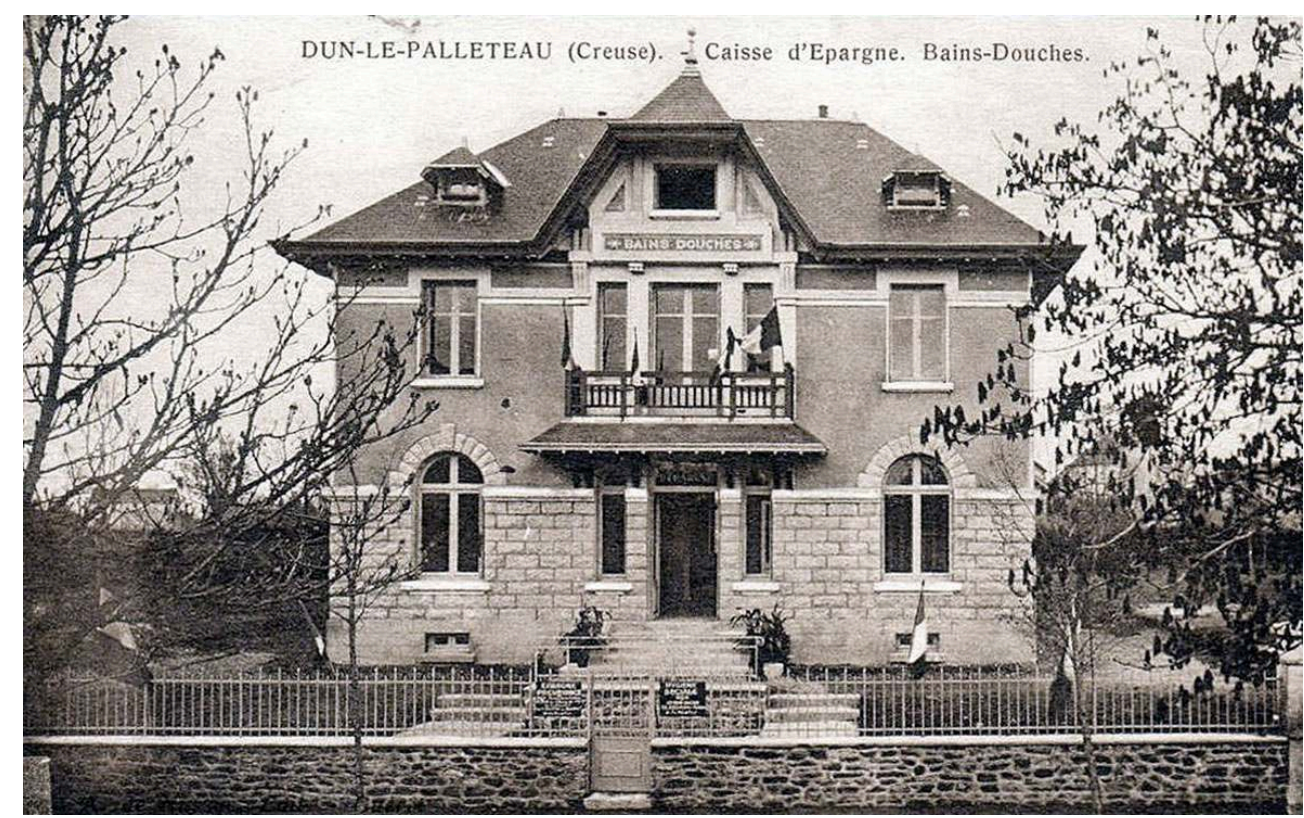

Bains-douches de Dun-le-Palestel, vue générale. Photothèque Paul Colmar.

(c) Photothèque Paul Colmar.

43 Toujours par souci d'économie et de rationalité, les bâtiments sont éclairés essentiellement en partie centrale par un lanterneau et les cabines sont équipées de fenêtres, aménagées en partie supérieure du mur afin de préserver l'intimité des usagers.

44 La caisse d'épargne souhaite ainsi, en construisant ces équipements modernes, s'intégrer dans le tissu local et devenir un acteur indispensable à la communauté.

\section{Une implantation inégale}

D'après nos recherches, aucun établissement de bains-douches du Limousin n'a été créé par des philanthropes ou des œuvres sociales privées, alors que ce fut le cas dans un grand nombre de régions. Seules quatre catégories de maîtres d'ouvrage sont représentées: les mutuelles, les communes, les caisses d'épargne et les habitations à bon marché (HBM).

\section{De fortes disparités géographiques}

La répartition géographique des différents établissements fait apparaitre de très fortes disparités entre les départements (fig. 12) selon le type de maîtres d'ouvrage. 
Figure 12

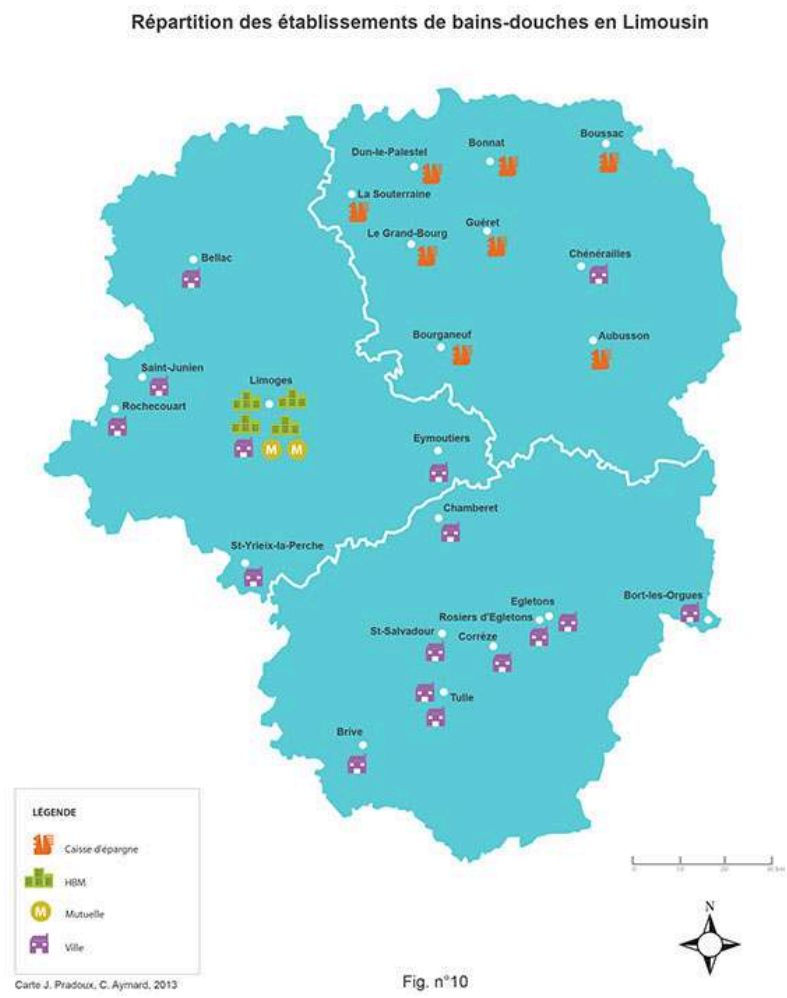

Répartition des bains-douches en Limousin.

Cartog. Pradoux, Josiane et Aymard, Colette. ( ) Josiane Pradoux et Colette Aymard.

l'établissement de Chénérailles est dû à une initiative communale. Pour expliquer cette " exception creusoise ", plusieurs hypothèses peuvent être envisagées mais aucune source ne vient les étayer :

49

- les caisses d'épargne de Guéret, de La Souterraine et d'Aubusson, avec leurs succursales, sont des organismes très actifs sur le plan social. Elles ne recourent à aucune une société intermédiaire pour financer l'œuvre sociale ; très dynamiques, elles veillent directement aux intérêts locaux ;

50 - la présence d'élites locales, particulièrement impliquées, et une solidarité communautaire très affirmée, pourrait expliquer la création volontariste de bainsdouches par la caisse d'épargne dans ce département. En devenant des membres du conseil d'administration des caisses d'épargne, les notables locaux agissent directement en faveur de l'intérêt public ;

51 - les maçons de la Creuse ont une forte tradition d'émigration, contrairement aux autres départements. Cette mobilité professionnelle est liée à une culture libérale et à une éducation très favorable à la libre entreprise et au dynamisme entrepreneurial. Cet état d'esprit contribue activement à la prise en main des problèmes locaux et à une action participative directe ou indirecte.

52 Ces trois observations, sans doute combinées, peuvent expliquer la construction de nombreux établissements par les caisses d'épargne dans ce département. Les 
municipalités se sont effacées devant le «dynamisme entrepreneurial » et la prise en main " communautaire ", à travers la caisse d'épargne, du destin villageois.

À l'opposé, en Corrèze, l'action des villes a été décisive. Là, les communes se sont appropriées l'hygiène publique et sont à l'origine de la construction de bains-douches. Le contexte politique est marqué par un très fort anticléricalisme et un soutien sans faille à la République depuis la période révolutionnaire. Sous la III ${ }^{e}$ République, ce particularisme perdure. Les instituteurs contribuent fortement à cette mentalité. La Corrèze est aussi peu touchée par l'industrialisation et bénéficie d'un important protectionnisme, mis en place notamment pour combattre la "grande dépression agricole» de l'extrême fin du $\mathrm{XIX}^{\mathrm{e}}$ siècle. Les municipalités symbolisent alors l'institution républicaine laïque et universaliste au service de la population. C'est probablement pour ces diverses raisons qu'elles furent les seules à construire des bains-douches, créant de ce fait une " exception corrézienne » en Limousin. Même si la Première Guerre mondiale met un peu à mal la puissance des radicaux et des radicauxsocialistes, avec en 1919 la victoire du Bloc national, le communisme progresse rapidement en milieu rural. Ainsi règne en Corrèze une vision politique essentiellement de gauche qui souhaite protéger les biens modestes au détriment des "gros propriétaires " tout en restant un mouvement sociologiquement conservateur. La mairie socialiste ou communiste en Corrèze adopte une action publique prioritairement sociale avec une politique urbaine volontariste, principalement dans les domaines de l'aménagement, de la voirie et surtout du logement. L'hygiène, la natalité, les personnes âgées et les chômeurs bénéficient de nombreuses aides. Cette politique découle de la première déclaration de principes du Parti socialiste, adoptée en $1905 \mathrm{au}$ congrès du Globe, qui vit la fondation de la Section française de l'Internationale ouvrière, et, après 1920, de la création de la section française de l'Internationale communiste. Le résultat se traduisit par la constitution de "municipalités-providence » ${ }^{61}$. Le « bien public » et l'hygiène étaient naturellement de la responsabilité des mairies, perçues comme une émanation de la volonté populaire.

En Haute-Vienne, les maîtres d'ouvrage sont plus diversifiés. Tous les cas de figure existent, hormis les créations à l'initiative de la caisse d'épargne. Limoges fait bien évidemment figure d'exception avec ses nombreux bains-douches aménagés par les HBM et les mutuelles. C'est une ville ouvrière dont les élus et acteurs locaux sont très attentifs aux questions sociales apparues au $\mathrm{XIX}^{\mathrm{e}}$ siècle, particulièrement celles liées à l'hygiène et à l'insalubrité des logements.

La cartographie des bains-douches permet de montrer la répartition équilibrée entre les trois départements ( 9 en Corrèze, 9 en Creuse et 14 en Haute-Vienne). Ils se trouvent essentiellement dans les grandes villes ou dans les villes chef-lieu de canton. Les bainsdouches sont donc considérés comme un équipement indispensable dès qu'un certain seuil administratif de la hiérarchie urbaine est atteint. Les grandes villes sont les premières pourvues. Guéret, puis Tulle, avec leur établissement de bains-douches datant des années 1907-1910, font figure de pionnières en Limousin.

\section{Un service public éphémère}

56 L'histoire des bains en Limousin diffère peu de celle des autres régions. Les premiers bains-douches apparaissent en Limousin après 1910 pour disparaître dans les années 1960, avec un pic à la fin de cette décennie. 

est celui de Guéret, financé par la caisse d'épargne. Viennent ensuite en 1910 les bains mutualistes de Limoges et les bains municipaux du pont de la Barrière à Tulle, puis en 1913, les bains-douches de Bourganeuf, bâtis par la caisse d'épargne.

1913 la société d'habitations à bon marché construit deux autres bains-douches à Limoges dans des quartiers à forte population ouvrière, comme les Marronniers ou Montjovis. Ces bains-douches sont devenus indispensables devant la forte urbanisation et la croissance importante du nombre d'ouvriers à la fin du XIX ${ }^{e}$ siècle. Et pourtant, à Limoges, l'hygiène n'est pas au centre des priorités municipales. Les notables limougeauds participant au conseil municipal sont favorables à une gestion parcimonieuse des fonds communaux ; l'hygiène n'apparaît pas encore comme un sujet prioritaire et est quelquefois considérée comme une dépense qui ne rapporte rien à la Ville. Le conseil municipal se préoccupe avant tout des ressources financières de la population ouvrière, touchée régulièrement par les crises porcelainières : il accorde des subventions pour soutenir quelques établissements de bienfaisance afin de limiter les effets du chômage et de la misère. La municipalité possède même parmi ses services un bureau de bienfaisance. Par ailleurs, la municipalité doit faire face à un sérieux problème de pénurie d'eau, à l'absence ou à la vétusté des canalisations et doit répondre à de nombreuses demandes d'installation de bornes-fontaines. Cependant, de 1876 à 1881, les réseaux d'approvisionnement en eau, la construction de nouveaux égouts, le pavage de nombreuses rues et la construction des trottoirs sont réalisés ${ }^{62}$. Les conduites d'eau sont remplacées de 1890 à 1900 . Ce n'est que tardivement, après la loi municipale de 1884 rappelant aux maires leur rôle en matière de salubrité publique, les mesures destinées à renforcer l'aide aux populations défavorisées ${ }^{63}$, et sous la mandature du préfet Charles Lallemand, de 1907 à $1909^{64}$, que l'hygiène sociale devient une priorité à Limoges. C'est sous la direction de ce nouveau préfet qu'est fondée la société anonyme "L'Étoile de Limoges", destinée à assurer la construction de «logements populaires, hygiéniques et économiques ${ }^{65}$ ». Elle compte parmi ses réalisations deux immeubles situés dans le faubourg Montjovis et le quartier des Marronniers. Le logement social est très contrôlé par le comité de patronage des HBM qui garantit la salubrité par des règles sanitaires très strictes.

Brive (Corrèze) est un cas un peu particulier : la construction de bains-douches a été planifiée en 1913 et retardée par le début des hostilités. Ces bains-douches, édifiés en fait de 1920 à 1924, ont une vocation bien particulière puisqu'ils sont liés à la construction d'une salle de gymnastique. Ils s'inséraient donc, avec le gymnase, dans un dispositif censé favoriser la préparation militaire et l'exercice physique.

De 1920 à la fin des années 1930, ce mouvement se poursuit grâce à divers maîtres d'ouvrage.

61 La construction de logements sociaux continue après la Grande Guerre, dans un contexte national favorable mais ralenti en raison de la crise du bâtiment et du coût exponentiel des matériaux; à Limoges, l'office municipal d'habitations à bon marché est créé en 1920 et la cité de Beaublanc est réalisée en 1924 avec des bains-douches. Désormais, plus aucun lotissement de logement social n'est construit sans bainsdouches. La cité des Couture créée en 1924, décidée dans l'esprit des cités-jardins par l'office d'HBM de Limoges, se veut exemplaire et intègre dans sa réalisation des bainsdouches parmi les équipements collectifs considérés alors comme essentiels tels qu'un lavoir et une garderie d'enfants. 

bains-douches, parallèlement à la création de logements sociaux. Cette tendance est plus marquée dans la Creuse et dans les villes ouvrières et manufacturières comme Aubusson, où la caisse d'épargne associe à un programme de construction de logements sociaux la construction d'un bâtiment de bains-douches. Dans le contexte ouvrier de la ville, la caisse d'épargne se préoccupe ainsi de fournir des logements sains et permet la pratique d'une meilleure hygiène des corps pour tous. Il semblerait aussi que l'on sousentende dans les conseils d'administration des caisses d'épargne que les villes chefslieux de canton devraient bénéficier d'équipements modernes en matière d'hygiène. C'est d'ailleurs un des arguments cité en faveur de la création de bains-douches, évoqué lors des débats en conseil (par exemple à Grand-Bourg ou Boussac, en Creuse). sur l'organisation communale imposent la construction de maisons-écoles (comme à Rosiers d'Égletons, Corrèze) et d'hôtels de ville et proposent aux communes d'importantes subventions. Les Villes en profitent pour équiper ces nouveaux bâtiments de bains-douches, considérés comme des services municipaux. Le regroupement de ces services (école, mairie, corbillard, lavoir, foyer familial, poste, bains-douches, etc.) ne se retrouve qu'en Corrèze (commune de Corrèze) et est symptomatique de la volonté de créer des bâtiments communaux utiles aux citoyens. Les bains-douches installés dans les mairies contribuent à asseoir la notion d'édifice public et à affirmer le rôle social de la municipalité. Ces édifices polyvalents sont créés dans les années 1920.

Les années 1920, 1930 et 1940 sont marquées par la loi Cornudet de 1919, qui favorise la réalisation de plans d'aménagement. La notion d'urbanisme se diffuse et le rôle des architectes prend davantage d'importance ${ }^{66}$. De nombreuses municipalités entreprennent alors d'importants aménagements pour répondre aux nouvelles conceptions urbaines. Quelques bains-douches sont édifiés dans ce contexte, comme à Saint-Yrieix-la-Perche, à Corrèze ou à Chamberet.

Parallèlement, à partir des années 1920, les villes modernisent leurs équipements et réseaux pour répondre à de nouvelles normes. Certes, la loi du 15 février 1902 relative à la protection de la santé publique ne prévoit rien pour le traitement des eaux usées, et l'assainissement des communes reste, en France, au tout début du siècle, très marginal. Frédéric Diénert dénombre seulement 18 villes ayant un système d'épuration des eaux sur tout le territoire, en $1924^{67}$. La majorité des communes du Limousin modernise ses réseaux publics et surtout celui d'eau potable dans les années 1920-1940. Ces travaux sont l'occasion pour les municipalités d'édifier des bains-douches, des fontaines et des lavoirs, comme à Rochechouart (Haute-Vienne), La Souterraine (Creuse), Bort-lesOrgues (Corrèze) et Égletons (Corrèze). La plupart des établissements sont aménagés alors qu'une grande majorité de logements sont dépourvus de salle de bain. Or les normes en matière d'hygiène et les besoins de la population dans ce domaine se développent.

Dans les années 1950 sont encore construits quelques bains-douches. Ce sont les derniers à voir le jour, malgré la modernisation des logements et les progrès des réseaux techniques urbains, comme à Saint-Junien et à Chénérailles. À Bellac est réalisée une autre construction tardive, de 1956-1958, à proximité du stade municipal. On est loin des notions initiales d'hygiène et désormais, c'est la pratique du sport qui réclame des douches. L'hygiène est désormais associée aux loisirs et au sport, 
démocratisés grâce aux congés payés, et symboles de plaisir et de bien-être. La construction de ces deux derniers bains-douches intervient alors que depuis les années 1950, les nouveaux logements sont équipés de salles de bain. De plus, dès les années 1960, les équipements intérieurs des anciens logements sont modernisés et les nouveaux sont adaptés aux normes de l'époque. Les salons des Arts ménagers des années 1950 contribuent à diffuser largement les nouvelles conceptions du confort et de l'hygiène à l'intérieur de l'habitat: chaque appartement est équipé d'une arrivée d'eau potable et d'une salle de bains. La machine à laver se généralise à cette période. Dans ce contexte, les bains-douches deviennent très rapidement obsolètes. La notion d'hygiène populaire laisse place à une conception plus individualiste et plus intimiste de l'hygiène. Cette arrivée du confort moderne dans les habitations françaises au cours des années 1960 sonne le glas des bains-douches.

\section{Conclusion}

67 Sans raison d'être, à partir du milieu du $\mathrm{XX}^{\mathrm{e}}$ siècle, les bains-douches ont été majoritairement reconvertis et leur installation sanitaire intérieure irrémédiablement détruite, l'architecture fonctionnelle de ces édifices les rendant difficilement réutilisables. Sur les 30 établissements répertoriés, 8 avaient disparu en 2009. Les autres, entièrement réaménagés, ont été affectés à un tout autre usage : bâtiments à vocation municipale ou sociale (ateliers, lieu de stockage, cantine scolaire, office de tourisme, salle d'exposition ou de sport...), espaces communs pour les habitats collectifs (salle commune pour des réunions, bureaux, foyer, salle de sport...), bâtiments à usage privé (rachat par des particuliers pour un réaménagement en lieu de vie). Seuls ceux de l'avenue Locarno, à Limoges, à proximité de la gare, sont encore en fonction mais leur surface a été réduite de moitié pour être mise à disposition d'un club sportif.

Marqueurs de la naissance de l'hygiène populaire, ces établissements ont favorisé la généralisation du concept du lavage entier du corps au sein de toutes les couches sociales et surtout des plus défavorisées. Les bains-douches ont largement contribué à l'amélioration des conditions sanitaires, des années 1910 à la fin des années 1960, au recul des épidémies et de diverses maladies et au progrès de la salubrité. Ils ont aussi participé à un certain nivellement des différences sociales tout comme le feront, par la suite, les nouvelles normes pour l'habitat dans les années 1950. En Limousin, les derniers bains-douches ont fermé dans les années 1980.

Pour ce qui est de leur protection au titre des monuments historiques, seuls ceux du pont de la Barrière à Tulle ont été jugés suffisamment remarquables pour bénéficier d'une inscription.au titre des monuments historiques. Dans cet exemple, l'architecte Joseph Auberty a mis en œuvre les règles de la composition traditionnelle avec des formes stylistiques d'esprit néo-classique en leur ajoutant un décor minimal d'inspiration Art nouveau (cercles, arcs, sculptures florales et végétales, etc.).

Cinq autres édifices ont été labellisés "patrimoine $d u x^{e}$ siècle»; ils sont représentatifs de la naissance du mouvement de l'hygiène publique en Limousin (Guéret, premier établissement en 1909), d'un style décoratif (bains-douches d'Aubusson avec son décor de briques vernissées de couleur), du style architectural des années trente (Rochechouart avec son pavillon central et ses deux terrasses) ou enfin de celui des années 1950 (Chénérailles avec ses bandeaux corniches et ses entablements en béton armé). 

chercheurs.

\section{NOTES}

1. - AYMARD, Colette (dir.). Les bains-douches en Limousin: architecture et histoire. Naissance d'une hygiène populaire. Limoges : DRAC du Limousin/CAUE de la Haute-Vienne, 2013.

2. - Labellisé « patrimoine du $\mathrm{Xx}^{\mathrm{e}}$ siècle ».

3. - Voir BARDIÈS-FRONTY, Isabelle, BIMBENET-PRIVAT, Michèle, WALTER, Philippe (dir.). Le bain et le miroir. Soins du corps et cosmétiques de l'Antiquité à la Renaissance. Cat. expo. Paris, musée de Cluny/Écouen, musée national de la Renaissance, 20 mai-21 septembre 2009. Paris : Gallimard, 2009.

4. - VIGARELLO, Georges. Le propre et le sale. L'hygiène du corps depuis le Moyen Âge. Paris : éd. du Seuil (Points), 1985, p. 174.

5. - CSERGO, Julia. La morale des corps. Le soin de propreté corporelle à Paris. Évolution des normes et des pratiques. 1850-1900. Thèse de sociologie, Paris, EHESS, 1986, p. 256.

6. - Vestiaires de planches, location de chemises et de serviettes, cordes tendues dans l'eau, etc.

7. - VIGARELLO, Georges. Op. cit., p. 204.

8. - À Paris, d'après Julia Csergo (thèse citée, p. 257-263), les établissements de bains froids se partagent entre les «bains à quatre sous » et les écoles de natation pour des classes sociales plus aisées.

9. - VIGARELLO, Georges. Op. cit., p. 19.

10. - En 1773, il y en a 9 à Paris. Certains pratiquent l'hydrothérapie médicale, d'autres sont des institutions de luxe avec concerts, collations, etc.

11. - VIGARELLO, Georges. Op. cit., p. 171 et 203 et CSERGO, Julia. Thèse citée, p. 257-274.

12. - Ce type de bain est déjà vanté à la fin du XVIII ${ }^{\mathrm{e}}$ siècle par Nicolas Roger : [FEYDEL, Gabriel]. Essai sur l'art de nager. Londres [Paris] : 1783.

13. - CSERGO, Julia. Thèse citée, p. 232-246. Les prix pratiqués peuvent être élevés et ces établissements ne sont souvent destinés qu'à une certaine classe de la population : DELABOST, Dr Merry. «Un établissement de bains-douches à bon marché à Rouen ». Extr. des Annales d'Hygiène publique et de médecine légale, 1898, p. 56.

14. - L'Almanach limousin y décrit en 1860 des rendez-vous galants.

15. - Conservé au musée du Louvre (département des Peintures, RF 1728).

16. - LÉVY, Michel. Traité d'hygiène publique et privée. Paris : J.-B. Baillière et fils, 1857 ( $3^{\mathrm{e}}$ éd.), t. II, p. 246 et suiv.

17. - Entre autres : DECHAMBRE, Amédée (dir.). Dictionnaire encyclopédique des sciences médicales. Paris: G. Masson/P. Asselin, 1869-1889, 100 vol.; TARDIEU, Ambroise. Dictionnaire d'hygiène publique et de salubrité. Paris : J.-B. Baillière et fils, 1852-1854, 3 vol.

18. - MÉNESTREL, Dr Paul (éd.), THOUVENEL, Dr Pierre Sébastien Barthélemy. Éléments d'hygiène. Paris : Baillière, 1840.

19. - FONSSAGRIVES, Jean-Baptiste. La maison. Étude d'hygiène et de bien-être domestiques. Montpellier : Gras, 1871, p. 209.

20. - VIGARELLO, Georges. Op. cit., p. 183. 
21. - Comme la prison de Rouen, de Lyon ou la prison de la Santé à Paris. En 1873, la prison de Rouen fit œuvre pionnière lorsque le médecin en chef, le docteur Merry Delabost, préconisa d'utiliser la pluie d'eau chaude pour entretenir la santé des prisonniers.

22. - La première crèche de Paris fut fondée le 14 novembre 1844 dans le quartier de Chaillot, quartier alors très pauvre. Les crèches étaient initialement des œuvres charitables destinées à des populations indigentes. Ce n'est qu'en 1862 que l'État réglementa leur fondation. Auparavant, elles étaient souvent créées sous le patronage de religieuses ou de femmes du monde.

23. - «...Sophie pensa qu'il était bon de laver les poupées, puisqu'on lavait les enfants... ». Dans SÉGUR, Sophie, comtesse de. Les malheurs de Sophie. Paris : Librairie Gedalge, 1858, (L. Hachette, (bibliothèque rose illustrée) 1859 ?) chap. sur la poupée de cire, p. 13-14. Noter que la comtesse de Ségur est également l'auteur de La santé des enfants. Paris : L. Hachette, 1857.

24. - Les maisons de poupée contemporaines sont souvent équipées d'un cabinet de toilette ou d'une salle de bains avec baignoire. Dans l'ouvrage Jeux et exercices de jeunes filles publié en 1856 par Mme de Chabreul, un chapitre traite de «La toilette de Madame " (première partie : "Jeux d'action ». Paris : L. Hachette, 1867 ? (3 éd.), p. 26-27).

25. - Conseil d'hygiène de Nantes, 1852, cité dans LÉONARD, Jacques. Les médecins de l'Ouest au XIX siècle. Thèse de lettres, Paris IV, 1975, p. 1142.

26. - LANEYRIE-DAGEN, Nadeije, VIGARELLO, Georges. La toilette. Naissance de l'intime. Cat. expo. Paris, musée Marmottan, 12 février-5 juillet 2015. Paris : Hazan/musée Marmottan Monet, 2015, p. 125.

27. - Voir par exemple MENIER, Dr H. M. Mon docteur. Traité de médecine et d'hygiène. Méthodes scientifiques et populaires. Paris: Librairie commerciale, 1907 (Grand Prix à l'Exposition internationale du livre, 1907), vol. 1, p. 441.

28. - CSERGO, Julia. Liberté, égalité, propreté. La morale de l'hygiène au XIX siècle. Paris : Albin Michel, 1988 , p. 78.

29. - GENCÉ, Comtesse de. Le cabinet de toilette d'une honnête femme. Paris: Bibliothèque des ouvrages pratiques, 1904.

30. - TRAMAR, Comtesse de. L'étiquette mondaine, usages de la société moderne dans toutes les circonstances de la vie. Paris : V. Havard, 1905.

31. - STAFFE, Blanche (baronne Staffe). Usages du monde. Règles du savoir-vivre dans la société moderne. Paris : V. Havard, 1891 (24 éd.).

32. - « tub»: cuvette servant aux ablutions du corps.

33. - Cité dans BONNEMAIN, Henri. «Un rapport sur la création de bains-douches municipaux à Vincennes ». Revue d'histoire de la pharmacie, 1969, vol. 57, n²02, p. 419-420.

34. - CSERGO, Julia. Liberté, égalité, propreté. Op. cit., p. 72.

35. - HAUSSER, A.-Édouard. "L'œuvre des bains-douches à bon marché ». Revue politique et parlementaire, Paris, juillet 1902, p. 7.

36. - ELEB, Monique. «La mise au propre en architecture. Toilette et salle de bains en France au tournant du siècle (1880-1914) ». Techniques \& culture, 2010, n54-55, Cultures matérielles, chap. VII « Techniques du corps et esthétique », p. 588-609.

37. - DEBOVE, Georges Maurice, PLICQUE, Albert-Faron. Hygiène. Paris : Ch. Delagrave, 1907.

38. - Rochard et Vallin décrivent ce système mis en place dans de nombreux établissements en Allemagne et à Vienne (p. 714-728). Ils vantent aussi le système par aspersion (p. 732-733) : «Ce sont les seuls qui satisfassent à la fois aux conditions qu'imposent l'hygiène et l'économie. [...] Les bains de mer et de rivière ne se prennent que dans l'été et tout le monde ne peut pas en faire usage : les bains de baignoire sont trop chers, les bains de piscine sont trop rares, tandis que l'aspersion permet de nettoyer un très grand nombre de personnes en très peu de temps, à très peu de frais et n'exige pas d'installation coûteuse... ». ROCHARD, Jules (dir.). Encyclopédie d'hygiène et de médecine publique. Paris : Lecrosnier et Babé, puis Babé et Cie, puis Bataille, puis A. Rousseau et Vigot, 1890-1897, t. 3, 1891. 
39. - Arch. dép. Haute-Vienne, cote $4 X 74$.

40. - CAZALET, Charles. L'œuvre des bains-douches à bon marché. Bordeaux : J. Durand, L. Delbrel et Cie successeurs, 1904, (extr. de la Revue politique et parlementaire, ${ }^{\circ} 126,1904$ ).

41. - DELABOST, Dr Merry. Op. cit., p. 1-10.

42. - « L'œuvre parisienne ».

43. - « L'œuvre rochelaise ».

44. - Désormais les sociétés qui construisent des bains-douches peuvent emprunter à taux réduit à des organismes publics : établissements charitables, Caisse des dépôts, caisses d'épargne.

45. - La "Société des équitables pionniers de Rochdale» : coopérative regroupant 28 ouvriers tisserands près de Manchester.

46. - Napoléon III les nomme « sociétés approuvées ».

47. - Ce système est le principe même de la sécurité sociale actuelle.

48. - Fermé en 1980, il a été détruit.

49. - Décret du 5 novembre 1926.

50. - Cet édifice est protégé au titre des monuments historiques. Voir dans la base Mérimée : notice PA19000027.

51. - Il est labellisé « patrimoine du $\mathrm{Xx}^{\mathrm{e}}$ siècle ».

52. - Cet établissement, également labellisé " patrimoine du $x^{e}$ siècle », a fonctionné jusqu'en 1983.

53. - D'après les plans de l'architecte Marcel Rauby.

54. - CAZALET, Charles. Art. cit., p. 14.

55. - Ce fut le cas à Marseille.

56. - À Lyon.

57. - CAZALET, Charles. Art. cit., p. 10.

58. - 1818 : date de la création de la première caisse d'épargne à Paris.

59. - Il a été labellisé « patrimoine du $\mathrm{xx}^{\mathrm{e}}$ siècle » par la DRAC Limousin.

60. - Sur 7,25 $\mathrm{m}$ de longueur.

61. - PAYRE, Renaud. Une science communale? Réseaux réformateurs et municipalité providence. Paris : CNRS Éditions, 2007.

62. - FRIOUX, Stéphane. La conquête de l'hygiène. Limoges. 1849-1914. Mémoire de maîtrise, histoire contemporaine, Lyon, université Jean-Moulin-Lyon 3, 2002, p. 162-163.

63. - Loi de 1893 sur l'assistance médicale gratuite ; loi de 1902 ; etc.

64. - Il ne reste que deux ans à Limoges mais y réalise un travail considérable en faveur de l'hygiène sociale. À ce titre, il contribue à stimuler les initiatives et à attirer l'attention sur les impératifs en matière d'hygiène, de prévention de la tuberculose, de soins médicaux, d'éducation populaire, salubrité des logements, etc.

65. - Arrêté ministériel du 31 décembre 1907 approuvant les statuts de la société.

66. - Voir les Congrès internationaux d'architecture moderne (CIAM) qui lancent les premières réflexions sur l'espace urbain et les pratiques urbanistiques. La charte d'Athènes fixe en 1933 les principales théories de l'urbanisme contemporain.

67. - DIENERT, Frédéric. "Épuration des eaux d'égout en France. État actuel de la question ». Revue d'hygiène et de police sanitaire, 1924, nº 46, p. 1096. 


\section{RÉSUMÉS}

La Conservation régionale des monuments historiques du Limousin a dressé un inventaire des bains-douches de la région. Ce corpus régional a été l'occasion de mener des recherches tant historiques qu'architecturales sur les bains populaires dits de "propreté ». Ces constructions fonctionnelles, souvent modestes, sont des témoins de l'évolution des pratiques de l'hygiène et de la société d'une époque désormais révolue. Communes, caisses d'épargne, sociétés d'habitations à bon marché et mutuelles en ont été les principaux maîtres d'ouvrages.

The historic monuments service in the Limousin region has recently carried out an inventory of the region's 'bains-douches', that is to say the public bathing and shower facilities. This regional corpus was an opportunity for carrying out some historical and architectural research on these popular bathing houses known as 'bains de propreté', or cleanliness baths. They were functional constructions, often of modest appearance and scale and they bear witness to evolving practices of hygiene in a social organisation that has now disappeared. The main providers of these bathing houses were local authorities, savings banks and low-cost housing associations.

\section{INDEX}

Keywords : hygiene, bathing houses, Limousin, caisse d'Epargne, savings bank, mutual aid societies, low-cost housing associations, commune, Creuse, Corrèze, Haute-Vienne

Mots-clés : hygiène, bains-douches, Limousin, Caisse d'épargne, œuvres mutualistes, Sociétés d'habitations à bon marché, commune, Creuse, Corrèze, Haute-Vienne

\section{AUTEUR \\ COLETTE AYMARD}

Conservateur des monuments historiques, DRAC Île-de-France, en poste en Limousin jusqu'au $1^{\mathrm{er}}$ janvier 2011 colette.aymard@culture.gouv.fr 